\title{
Feasibility report of conservative surgery, perioperative high-dose-rate brachytherapy (PHDRB), and low-to-moderate dose external beam radiation therapy $(\mathrm{EBRT})$ in pediatric sarcomas
}

Rafael Martínez-Monge ${ }^{1}$ * Cristina Garrán ${ }^{1}$, Mauricio Cambeiro ${ }^{1}$, Mikel San Julián ${ }^{2}$, Juan Alcalde $^{3}$, Luis Sierrasesúmaga ${ }^{4}$

${ }^{1}$ Department of Oncology, University of Navarra Clinic, University of Navarra, Pamplona, Spain

${ }^{2}$ Department of Orthopedic Surgery, University of Navarra Clinic, University of Navarra, Pamplona, Spain

${ }^{3}$ Department of Head and Neck Surgery, University of Navarra Clinic, University of Navarra, Pamplona, Spain

${ }^{4}$ Department of Pediatrics, University of Navarra Clinic, University of Navarra, Pamplona, Spain

* Corresponding author. Department of Oncology, Clínica Universitaria de Navarre, University of Navarre, Avda Pío XII s/n Pamplona, Navarre, Spain.

Tel.: +34-948-255400; fax: +34-948-255500.

E-mail address: rmartinezm@unav.es (R. Martínez-Monge).

\section{ABSTRACT}

Purpose: This study was undertaken to determine the feasibility of perioperative highdose-rate brachytherapy (PHDRB) as an accelerated boost in patients with pediatric sarcomas.

Methods and materials: Five pediatric patients (ages 7-16) with soft tissue sarcomas (STS) or soft tissue recurrences of previously treated osteosarcomas were treated with surgical resection and PHDRB (16-24 Gy) for R0-R1 resections. Patients with STS and osteosarcomas received 27 Gy and 45 Gy of EBRT postoperatively.

Results: After a median follow-up of 27 months (range, 12-50) all the patients remain locally controlled. Only 1 patient developed regrowth of pulmonary metastases and died of distant disease at 16 months.

Conclusions: The use of PHDRB is safe in the short-term in this pediatric population. Only 1 patient suffered a partial wound dehiscence that may not be entirely related to PHDRB. Patients withrecurrent osteosarcomas canbe treatedinafashion similartotheir adultsoft tissuecounterpartsand avoid limb amputation. Younger patients with STS may achieve local control and prevent growth retardation with a combination of PHDRB and moderate doses of EBRT.

Keywords: Pediatric; Soft tissue sarcomas; Osteosarcoma; Perioperative; High dose rate; Brachytherapy 


\section{INTRODUCTION}

Conventional EBRT is an integral part of the treatment of most children and adolescents with soft tissue sarcomas (STS). However, in the growing child, potential growth retardation and deformity associated with the use of EBRT are a serious concern that can limit its use (1).

Radiation techniques that improve the therapeutic ratio are especially important for these growing individuals. Intraoperative radiation boosting techniques have the advantage of direct tumor bed localization and displacement of radiosensitive structures, where possible, away from the area irradiated. The majority of worldwide experience has been with LDR manual brachytherapy, and this modality has produced acceptable local control and complication rates (2-4). The major disadvantages with this modality have been the issue of radiation protection, the need to immobilize and sedate the younger children during treatment, and the psychological trauma associated with separation from the parents. The advent of remote-controlled LDR, PDR, and HDR machines as well as the development of intraoperative high-dose-rate brachytherapy (IOHDR) and intraoperative electron beam radiotherapy (IOERT) has eliminated radiation exposure hazards to the patient's family and medical personnel.

IOHDR and IOERT have been used in pediatric patients (5-7). However, high-dose ( $\geq 15$ Gy) IOERT has been associated with clinically significant toxicity in late-reacting tissues such as vessels and nerves present in the surgical bed (8). As a consequence, these normal tissues at risk of harboring metastatic deposits may remain undertreated for fear of long-term toxicity, and this reluctance to treat may ultimately lead to treatment failure.

High-dose-rate (HDR) brachytherapy alone has been used with success in the younger patients with more chemoresponsive tumors (e.g., rhabdomyosarcoma) in whom the smaller postchemotherapy tumor volume can be encompassed with an interstitial or intracavitary implant, eliminating the need for additional EBRT (9). However, older children with more extensive tumors cannot be treated with brachytherapy alone and may require EBRT.

For patients requiring EBRT, we designed a treatment protocol that involved using perioperative HDR brachytherapy (PHDRB) and postoperative low-to-moderate dose EBRT (27-45 Gy). The outcomes for patients treated by PHDRB are reported in this article.

\section{METHODS AND MATERIALS}

From June 2000 to August 2003, 5 patients with pediatric sarcomas (4 boys, 1 girl; ages ranging from 7 to 16 years; median, 11.0 years) were treated with PHDRB and conservative surgery. The PHDRB dose was 4 Gy b.i.d. (interfraction interval of at least 6 h) X 6 (24 Gy total dose). Radiation treatment was completed with EBRT (27-45 Gy in 15-25 treatments, 1.8 Gy daily fractions) $4-5$ weeks after the surgical procedure. The lower dose was used for the younger patients with soft tissue sarcomas, and the higher dose was used in the older patients with soft tissue recurrences of osteosarcomas. Total 
radiation doses equivalent to standard fractionation regimens delivered with 2 Gy daily fractions and calculated with the linear quadratic formulation without time correction (i.e., $B E D=n d[1+d /(\alpha / \beta)])(10)$ are shown in Fig. 1 .

The surgical and the radiation oncology teams used the preoperative physical and imaging, surgical findings, frozen sections where necessary, and gross examination of the surgical specimen to jointly determine the area to be implanted, although in most cases the entire surgical bed was implanted.

\section{Brachytherapy technique}

Traditional implant guidelines recently released by the American Brachytherapy Society were generally followed (11). The CTV was covered with a set of plastic catheters placed as near to parallel as possible at $1.0-1.5 \mathrm{~cm}$ intervals with a margin of $1 \mathrm{~cm}$ in all directions. A single plane implant array was the most frequently used. The lateral margins were treated with additional catheters extending beyond the CTV. The longitudinal margins were added by setting the distal and proximal ends of the catheters at $1.5-2.0 \mathrm{~cm}$ beyond the skin incision. Catheters were inserted no more than $5 \mathrm{~mm}$ into the tumor bed to avoid underdosage of the surgical surface. This maneuver avoided catheter displacement and resulted in improved geometry. In cases in which the catheters could not be inserted below the surface, resorbable sutures were used to secure the catheters onto the surgical surface. If necessary, gelfoam or muscle flaps no more than $5 \mathrm{~mm}$ thick were interposed between the catheter plane and radiosensitive structures. Finally, catheters were attached at the skin exit points with plastic buttons and silk sutures. Once the implant was performed, the surgical team proceeded with the closure or, if necessary, with the reconstruction of the surgical defect with regional or microvascular free flaps.

\section{D dosimetry guidelines}

A CT scan for verification was performed during the first $48 \mathrm{~h}$ after surgery, once the patient was in stable condition and ready for transportation. The CT study was transferred to the Radiation Treatment Planning System for dosimetry. The treatment planning process followed the Paris System with manual optimization for each of the CT slices. Additional rules included that the CTV be encompassed by the 4 Gy isodose line, defined as the minimum tumor dose (MTD) isodose line as per the ICRU No. 58 recommendations (12). An MTD to mean central dose (MCD) ICRU No. 58 ratio of not less than $70 \%$ was accepted, provided that the volume encompassed by the 6 Gy isodose (V150) was not greater than $50 \%$ of the volume encompassed by the 4 Gy isodose (V100). Automatic optimization algorithms provided by the Treatment Planning System were not allowed. Once the treatment plan was approved, it was transferred to the afterloader unit control console where the dwell positions and treatment times were double-checked by the radiation technologist. Brachytherapy delivery began 3-6 days after surgery (median, 5 days). The rest of the brachytherapy parameters are detailed in Table 1. 


\section{External radiation}

All patients underwent customized immobilization and virtual CT simulation before starting external beam therapy.

The CTV included the presurgical gross tumor extent with $5 \mathrm{~cm}$ margins in the direction of the body axis and $2-3 \mathrm{~cm}$ laterally as well as the entire surgical scar.

\section{Outcome analysis}

Local failure was defined as the occurrence of tumor regrowth in the area treated with brachytherapy or in an adjacent region (i.e., recognition failure). Any other failure in the anatomical region implanted but not in close continuity with the implanted area was considered as regional, and any failure elsewhere was considered distant failure.

Early toxicities were defined as those occurring from the date of surgery to 60 days after the completion of the treatment. Toxicities were classified as late if they occurred more than 60 days after the completion of the treatment. Toxicities were documented according to the Radiation Therapy Oncology Group (RTOG) morbidity scoring criteria.

\section{RESULTS}

After a median follow-up of 27 months (range, 12-50 months), all the patients remained locally controlled. In addition, 4 of 5 patients are alive and without evidence of disease. The other patient, with stage IV condroblastic osteosarcoma developed regrowth of pulmonary metastases at 9 months and died at 16 months without evidence of locoregional failure. Patient characteristics and outcome are shown in Tables 2 and 3. The following cases illustrate the possible usefulness of PHDRB in these specific disease situations.

\section{Case No. 1 (F.J.R.R.)}

This 11-year-old boy had been initially treated in 1999 with preoperative chemotherapy, surgery, and adjuvant chemotherapy according to the T-10 protocol (high dose methotrexate, bleomycin, cyclophosphamide, dactinomycin, cisplatin, and doxorubicin). The patient developed a wound dehiscence and multiple episodes of allograft infection during the administration of the adjuvant chemotherapy regimens, which led to several reoperations including two allograft replacements. He developed a local relapse involving the tibial allograft with soft tissue extension into the anterior aspect of the knee 14 months after the primary surgery. The MRI showed a lesion of $3.5 \mathrm{~cm}$ in diameter with some satellite nodules. He was operated on with a marginal resection of the lesion leaving close margins $(1 \mathrm{~mm})$. Three catheters were inserted into the deepest aspect of the tumor bed. The patient developed a partial wound dehiscence and surgical bed infection that led to the placement of a knee prosthesis 11 months later. The patient is alive and free of disease 50 months later and walks with the aid of crutches. 


\section{Case No. 2 (J.F.S.)}

This 16-year-old boy had been initially treated in 1999 with preoperative chemotherapy, surgery, and adjuvant chemotherapy according to the T-10 protocol in another institution. He developed pulmonary metastases and a local relapse in the poplyteal fossa 10 months after the primary surgery. The MRI showed a bulky soft tissue mass of $8.0 \mathrm{~cm}$ in diameter displacing the poplyteal vessels. Marginal resection of the lesion left close margins at the level of the poplyteal vessels $(7 \mathrm{~mm})$. Three catheters were inserted into the deepest aspect of the tumor bed. The patient died 16 months later after regrowth of pulmonary metastases and new onset brain metastases, although the tumor was locally controlled and the patient was fully ambulant.

\section{Case No. 3 (C.T.B.)}

This 11-year old girl presented with a $10 \mathrm{~cm}$ lump deep in the anterior compartment of the distal thigh, in proximity to the femoral bone. A conservative resection was carried out, and four afterloading catheters were placed onto the anterior aspect of the femur due to the concerns of inadequate resection margins at that level. The final pathology report revealed close margins $(6 \mathrm{~mm})$ at that level, and adding PHDRB and low-dose EBRT was recommended. No adjuvant chemotherapy was given. She is alive with fully preserved limb function and without evidence of disease at 34 months after surgery.

\section{Case No. 4 (A.L.L.)}

This 9-year old boy presented with a $4.5 \mathrm{~cm}$ mass infiltrating the pterygoid space in the vicinity of the carotid vessels and attached to the pterygoid process in the left side. He was initially treated with one course of neoadjuvant chemotherapy without remarkable response. A limited surgical resection left positive margins in the pterygoid process. Four catheters were placed for PHDRB. No adjuvant chemotherapy was given. The patient is alive with fully preserved oral aperture and masticatory function and without evidence of disease 12 months after surgery.

\section{Case 5 (D.F.O.)}

This 7-year old boy presented with a 1-cm mass involving the synovial capsule in the lateral aspect of the elbow. An excisional biopsy at another hospital demonstrated an unsuspected undifferentiated sarcoma. A reoperative procedure was recommended, and 4 catheters were left in place for PHDRB. Although the surgical specimen obtained demonstrated no tumor, complementing the surgical procedure with adjuvant PHDRB and low-dose EBRT was recommended due to the concerns about contamination of the surgical bed during the primary procedure. No adjuvant chemotherapy was given. Dose volume histograms revealed that the D10 $(10 \%$ of the volume of interest that receives the higher dose) of the distal (2.35 Gy) and proximal (3.00 Gy) growth plates of the elbow joint were below the prescription isodose of $4 \mathrm{~Gy}$. The patient is alive with fully preserved elbow motion and without evidence of disease 12 months after surgery. 


\section{DISCUSSION}

Comparison among different brachytherapy series on pediatric STS is especially difficult due to the small number of patients treated, the different brachytherapy modalities used (low dose rate, pulsed dose rate, fractionated and intraoperative high dose rate) and the use or not of EBRT. In addition, the inclusion of a relatively large percentage of patients with rhabdomyosarcoma, a more chemo- and radio-responsive pediatric sarcoma, may contaminate the final results obtained.

Dose guidelines for pediatric non-rhabdomyosarcoma STS are not clearly defined. We decided to treat the 3 patients of this disease category (patient 3, 4, and 5) with 24 Gy of PHDRB followed by 27 Gy of EBRT (Fig. 1). According to the LQ model (10) this is roughly equivalent to a total MTD of 55 Gy delivered with standard fractionation. Similar total doses had already been used in the management of pediatric STS by several institutions with excellent local control results $(4,13)$. The Institute Gustave Roussy, France (13), treated 127 children (mostly with STS) with LDR brachytherapy $(0.4-0.6 \mathrm{~Gy} / \mathrm{h})$ from 1972 to 1990 . Brachytherapy doses were 45-65 Gy when used as a sole modality and 15-25 Gy when used as a boost. Patients who received brachytherapy as primary treatment had local control of $81 \%$.

The addition of supplemental EBRT is also a matter of controversy due to the fear of long-term complications related to the use of this radiation modality in younger children (1). Nag et al. (9) from The Ohio State University published their results on 12 children with STS (9 rhabdomyosarcomas) with a median age of 18 months at diagnosis. Teletherapy was avoided in this young patient population. HDR brachytherapy was delivered in 3 Gy fractions twice a day to a total dose of 36 Gy in 8 days. Results showed a 6 year actuarial local control of $91 \%$. However, the use of brachytherapy alone raises the concern of decreased control rates due to the emergence of marginal failures, especially in non-rhabdomyosarcoma STS. Goodman et al. (7) reported the experience of the MSKCC with IOHDR in pediatric patients (mostly neuroblastoma, rhabdomyosarcoma, and Ewing's/PNET). A total of 66 pediatric patients with a median age of 7 years were reviewed. Fifty-three percent of the patients were treated for recurrent disease. Twentynine patients (44\%) received IOHDR (median, 12 Gy) and EBRT. The 2-year local control rate was 56\%. Postoperative EBRT significantly improved the 2-year local control rate from $29 \%$ to $83 \%(\mathrm{p}=0.002)$. According to the authors, the benefit of adjuvant EBRT after brachytherapy may be related to a doseresponse effect or better coverage of microscopic residual disease with the larger treatment volumes used for EBRT. In a similar approach, Nag et al. (5) combined a single dose of 10-12.5 Gy HDR given intraoperatively with modest doses (27-36 Gy) of teletherapy given postoperatively. Six children treated with this protocol are currently alive, and five are without evidence of disease after 40 months (range, 22-62 months).

Dose guidelines for pediatric osteosarcoma are not available. The use of adjuvant radiation therapy has been largely abandoned due to the extremely low local failure rates after neoadjuvant chemotherapy and surgical resection. Therefore, we extrapolated the National Comprehensive Cancer Center recommendations for adult STS with positive margins that call for 20 Gy of brachytherapy followed by 50 Gy of EBRT and treated the 2 patients of this disease category (patients 1 and 2) with 24 Gy of PHDRB followed by 45 Gy of EBRT (Fig. 1). 


\section{CONCLUSION}

The use of PHDRB is a feasible alternative in those patients in whom extensive surgery is inadvisable, and therefore more limited surgery is attempted to prevent long-term disability. The initial results show that a reasonable local control and long-term diseasefree survival can be achieved, despite a lower EBRT dose in those patients with nonrhabdomyosarcoma STS. In addition, those patients with recurrent osteosarcomas can attain long-term local control and avoid amputation. Although some major centers favor the use of HDR brachytherapy alone in the pediatric population, we preferred to use PHDRB in combination with EBRT due to preliminary data from The Memorial Sloan-Kettering Cancer Center (14) suggesting a trend in favor of brachytherapy boost combined with EBRT in patients with positive resection margins. Toxicity directly associated with radiation therapy has been modest even with the use of total doses that may exceed effective adult sarcoma doses in those patients treated for recurrent osteosarcoma. We have only observed one case of wound dehiscence that can be regarded as multifactorial in origin in view of the bizarre previous history. Long-term results in terms of local control and growth preservation must wait until the patients stop growing after puberty.

\section{REFERENCES}

1]. Paulino AC. Late effects of radiotherapy for pediatric extremity sarcomas. Int J Radiat Oncol Biol Phys 2004;60:265-274.

2]. Gerbaulet A, Panis X, Flamant F, et al. Iridium afterloading curietherapy in the treatment of pediatric malignancies. The Institut Gustave Roussy experience. Cancer 1985;56:1274-1279.

3]. Healey EA, Shamberger RC, Grier HE, et al. A 10-year experience of pediatric brachytherapy. Int J Radiat Oncol Biol Phys 1995;32: 451-455.

4]. Merchant TE, Parsh N, del Valle PL, et al. Brachytherapy for pediatric softtissue sarcoma. Int J Radiat Oncol Biol Phys 2000;46:427-432.

5]. Nag S, Martinez-Monge R, Ruymann FB, et al. Feasibility of intraoperative high-dose-rate brachytherapy to boost low-dose external beam radiation therapy to treat pediatric soft tissue sarcomas. Med Pediatr Oncol 1998;31:7985.

6]. Nag S, Retter E, Martinez-Monge R, et al. Feasibility of intraoperative electron beam radiation therapy in the treatment of locally advanced pediatric malignancies. Med Pediatr Oncol 1999;32:382-384.

7]. Goodman KA, Wolden SL, LaQuaglia MP, et al. Intraoperative high-dose-rate brachytherapy for pediatric solid tumors: A 10-year experience. Brachytherapy 2003;2:139-146.

8]. Wallace HJ, Willett CG, Shellito PC, et al. Intraoperative radiation therapy for locally advanced recurrent rectal or rectosigmoid cancer. J Surg Oncol 1995;60:122-127.

9]. Nag S, Martinez-Monge R, Ruymann F, et al. Innovation in the management of soft tissue sarcomas in infants and young children: High-dose-rate brachytherapy. J Clin Oncol 1997; 15:3075-3084. 
10]. Douglas BG, Fowler JF. The effect of multiple small doses of X-rays on skin reactions in the mouse and a basic interpretation. Radiat Res 1976;66:401426.

11]. Nag S, Shasha D, Janjan N, et al. The American Brachytherapy Society recommendations for brachytherapy of soft tissue sarcomas. Int J Radiat Oncol Biol Phys 2001;49:1033-1043.

12]. International Commission on Radiation Units and Measurements. Dose and Volume Specification for Reporting Interstitial Therapy. ICRU Report 58. Bethesda, MD: International Commission on Radiation Units and Measurements; 1997.

13]. Nag S, Gerbaulet A, Lartigau E, et al. Pediatric tumors. In: Nag S, editor. Principles and Practice of Brachytherapy. Armonk, NY: Futura Publishing Co.; 1997:497-610.

14]. Alekhteyar KM, Leung DH, Brennan MF, et al. The effect of combined external beam radiotherapy and brachytherapy on local control and wound complications in patients with high-grade soft tissue sarcomas of the extremity with positive microscopic margin. Int JRadiat Oncol Biol Phys 1996;36:321324. 
Table 1. Brachytherapy parameters

\begin{tabular}{|c|l|}
\hline Number of catheters & \\
\hline Median & 4 \\
\hline Range & $3-4$ \\
\hline Surgery to brachytherapy gap (days) & \\
\hline Median & 5 \\
\hline Range & $3-6$ \\
\hline Brachytherapy duration (days) & \\
\hline Median & 3 \\
\hline Range & 3 \\
\hline TV 100 (cc) & \\
\hline Median & 33,3 \\
\hline Range & $13,0-86,6$ \\
\hline TV 150 (cc) & \\
\hline Median & 12,6 \\
\hline Range & $4,8-35,1$ \\
\hline
\end{tabular}

$\mathrm{TV}_{100}=$ Tissue volume encompassed by the $100 \%$ prescription isodose (4Gy).

$\mathrm{TV}_{150}=$ Tissue volume encompassed by the $150 \%$ prescription isodose (6Gy). 
Table 2. Tumor and treatment characteristics

\begin{tabular}{|c|c|c|c|c|c|c|c|}
\hline $\begin{array}{c}\text { Case } \\
\text { no. }\end{array}$ & Histology & $\begin{array}{c}\text { AJCC } \\
\text { stage }\end{array}$ & Age & PHDRB site & $\begin{array}{c}\text { Resection } \\
\text { margins }\end{array}$ & $\begin{array}{c}\text { PHDRB } \\
\text { dose }\end{array}$ & $\begin{array}{c}\text { EBRT } \\
\text { dose }\end{array}$ \\
\hline 1 & $\begin{array}{c}\text { Osteoblastic } \\
\text { osteosarcoma }\end{array}$ & Recurrent & 11 & $\begin{array}{c}\text { Poplyteal } \\
\text { fossa }\end{array}$ & $1 \mathrm{~mm}$ & $24 \mathrm{~Gy}$ & $45 \mathrm{~Gy}$ \\
\hline 2 & $\begin{array}{c}\text { Condroblastic } \\
\text { osteosarcoma }\end{array}$ & Recurrent & 16 & $\begin{array}{c}\text { Poplyteal } \\
\text { fossa }\end{array}$ & $6 \mathrm{~mm}$ & $24 \mathrm{~Gy}$ & $45 \mathrm{~Gy}$ \\
\hline 3 & Synovial sarcoma & IIa & 11 & Thigh & $7 \mathrm{~mm}$ & $24 \mathrm{~Gy}$ & $27 \mathrm{~Gy}$ \\
\hline 4 & Fibrosarcoma & Ib & 9 & $\begin{array}{c}\text { Pterygoid } \\
\text { fossa }\end{array}$ & Positive & $24 \mathrm{~Gy}$ & $27 \mathrm{~Gy}$ \\
\hline 5 & $\begin{array}{c}\text { Undifferentiated } \\
\text { sarcoma }\end{array}$ & IIb & 7 & Elbow & Negative & $24 \mathrm{~Gy}$ & $27 \mathrm{~Gy}$ \\
\hline
\end{tabular}

\begin{tabular}{|c|c|c|c|c|c|c|}
\hline \multicolumn{7}{|c|}{ Table 3. Patient outcome } \\
\hline $\begin{array}{c}\text { Case } \\
\text { no. }\end{array}$ & Histology & Complications & $\begin{array}{c}\text { Local } \\
\text { control }\end{array}$ & $\begin{array}{c}\text { Distant } \\
\text { metastases }\end{array}$ & $\begin{array}{c}\text { Survival } \\
\text { (months) }\end{array}$ & Status \\
\hline 1 & $\begin{array}{c}\text { Osteoblastic } \\
\text { osteosarcoma }\end{array}$ & $\begin{array}{c}\text { Wound dehiscence } \\
\text { grade } 4\end{array}$ & Yes & No & $50+$ & NED \\
\hline 2 & $\begin{array}{c}\text { Condroblastic } \\
\text { osteosarcoma }\end{array}$ & None & Yes & Yes & 16 & DOD \\
\hline 3 & $\begin{array}{c}\text { Synovial sarcoma } \\
4\end{array}$ & None & Yes & No & $34+$ & NED \\
\hline 5 & $\begin{array}{c}\text { Undifferentiated } \\
\text { sarcoma }\end{array}$ & None & Yes & No & $12+$ & NED \\
\hline \multicolumn{7}{|c|}{ NED $=$ Alive, no evidence of disease; DOD = Dead of disease. } \\
\hline
\end{tabular}




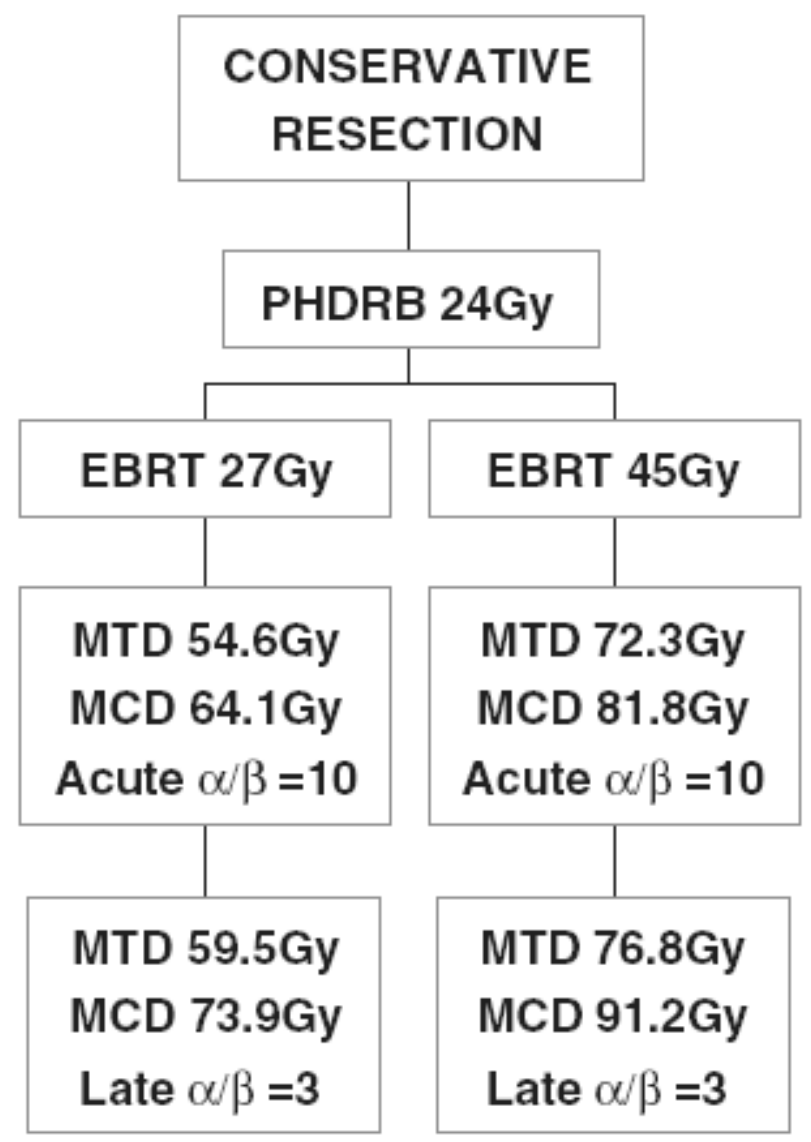

Figure 1. Treatment scheme. 